\title{
A RECURSIVE PROCEDURE FOR CALCULATION OF SOME COMPOUND DISTRIBUTIONS
}

\author{
By Ole Hesselager \\ University of Copenhagen
}

\begin{abstract}
We consider compound distributions where the counting distribution has the property that the ratio between successive probabilities may be written as the ratio of two polynominals. We derive a recursive algorithm for the compound distribution, which is more efficient than the one suggested by PANJER \& Willmot (1982) and Willmot \& Panjer (1987). We also derive a recursive algorithm for the moments of the compound distribution. Finally, we present an application of the recursion to the problem of calculating the probability of ruin in a particular mixed Poisson process.
\end{abstract}

\section{KEYWORDS}

Recursions; compound distributions; moments; probablity of ruin.

\section{INTRODUCTION}

Let

$$
X=\sum_{i=1}^{N} Y_{i}
$$

denote the aggregate claims amount where $X=0$ if $N=0$. It is assumed that the severities $Y_{1}, Y_{2}, \ldots$ are mutually independent and distributed on the non-negative integers with common probability function

$$
f_{y}=\mathrm{P}\left(Y_{i}=y\right), \quad y=0,1, \ldots
$$

It is further assumed that $N$ is stochastically independent of $Y_{1}, Y_{2}, \ldots$ with probability function

$$
p_{n}=\mathrm{P}(N=n), \quad n=0,1, \ldots
$$

The compound distribution

$$
g_{x}=\sum_{n=0}^{\infty} p_{n} f_{x}^{* n}
$$


where $f^{* n}$ denotes the $n$-th convolution of $f$, can sometimes be calculated recursively. PANJER (1981) derived his by now famous recursive formula for the case where the counting probabilities $p_{n}$ satisfy the recursive relation

$$
p_{n}=\frac{a n+b}{n} p_{n-1}, \quad n=1,2, \ldots
$$

SundT \& Jewell (1981) showed that (1.3) is satisfied by the Poisson, the binomial, and the negative binomial distributions, and no other. PANJER \& WILLMOT (1982) went on to consider the class of counting distributions which satisfy a recursion

$$
p_{n}=\frac{\sum_{i=0}^{k} a_{i} n^{i}}{\sum_{i=0}^{k} b_{i} n^{i}} p_{n-1}, \quad n=1,2, \ldots,
$$

for some $k$, and derived recursions for the compound distribution when $k=1$ and $k=2$. These recursions were further developed by WILlmot \& PANJER (1987). Recursions for a different extension of the class (1.3) can be found in SCHRÖTER (1990) and Sundt (1992).

In the case of arbitrary $k$, it is clearly not possible to give a complete characterization of the class (1.4). ORD (1967) characterizes those distributions which satisfy a difference equation analogous to Pearson's differential equation, and also derives a recursive relation for the (factorial) moments. Also GULDBERG (1931) considered recursive calculation of moments for certain members of the class (1.4).

Important distributions satisfying (1.4), which are not already covered by (1.3), are the hypergeometric distribution $(k=2)$, the Polya-Eggenberger distribution $(k=2)$, the Waring distribution $(k=1)$, and the generalized Waring distribution $(k=2)$.

Note that the coefficients $a_{i}$ and $b_{i}$ appearing in (1.4) are only specified up to a multiplicative constant.

In this paper we consider the class (1.4) and derive a new recursion for the compound distribution (Section 2). The derivation is elementary, and is valid for arbitrary $k$. In Section 3 we derive a recursion for the moments of the compound distribution. In Section 4 the proposed recursive formula for the compound distribution is compared to that of WILLMOT \& PANJER (1987) for $k=1$ and $k=2$, and is found to be more efficient. In Section 5 we present an application of the recursion to problem of calculating the probability of eventual ruin in a (particular) mixed Poisson process. 
2. RECURSION FOR THE COMPOUND DISTRIBUTION

Assume that $p_{n}$ satisfies (1.4). For $i=0, \ldots, k$ we define the auxiliary functions

$$
g_{i, x}=\sum_{n=0}^{\infty} n^{i} p_{n} f_{x}^{* n}, \quad x=0,1, \ldots,
$$

and note in particular that $g_{0, x}$ is the compound distribution (1.2). Let

$$
g_{x}=\left(g_{0, x}, \ldots, g_{k, x}\right)^{\prime},
$$

and let $m$ denote the smallest integer for which $f_{m}>0$. Thus, $f_{y}=0$ for $y=0, \ldots, m-1$. The following result gives a recursion for the vector $g_{x}$, and hence the compound distribution $g_{0, x}$.

Theorem 1: Assume that (1.4) holds true. With initial values

$$
\begin{aligned}
& g_{i, 0}=\sum_{n=0}^{\infty} p_{n} n^{i} f_{0}^{n}, \quad i=0, \ldots, k, \\
& g_{i, x}=0, \quad i=0, \ldots, k, \quad x=1, \ldots, m-1,
\end{aligned}
$$

the compound distribution $g_{x}=g_{0, x}$ may be obtained by calculating $g_{x}$ recursively as

$$
g_{x}=\boldsymbol{T}_{x}^{-1} \boldsymbol{t}_{x}, \quad x \geq m \vee 1,
$$

where

(2.4) $\quad \boldsymbol{T}_{x}=\left(\begin{array}{ccccc}1 & -m / x & 0 & \ldots & 0 \\ 0 & 1 & -m / x & \ldots & 0 \\ \vdots & \vdots & & & \vdots \\ 0 & 0 & \ldots & 1 & -m / x \\ \left(b_{0}-f_{0} c_{0}\right) & \left(b_{1}-f_{0} c_{1}\right) & \ldots & \left(b_{k-1}-f_{0} c_{k-1}\right) & \left(b_{k}-f_{0} c_{k}\right)\end{array}\right)$,

and $\boldsymbol{t}_{x}=\left(t_{0, x}, \ldots, t_{k, x}\right)^{\prime}$ is given by

$$
\begin{aligned}
& \text { (2.5) } t_{i, x}=\frac{1}{f_{m}} \sum_{y=1}^{x} f_{m+y}\left\{\frac{m+y}{x} g_{i+1, x-y}+\frac{y-x}{x} g_{i, x-y}\right\}, \quad i<k, \\
& \text { (2.6) } t_{k, x}=\sum_{y=m \vee 1}^{x} f_{y} \sum_{i=0}^{k} c_{i} g_{i, x-y},
\end{aligned}
$$

with $c_{i}=\sum_{j=i}^{k}\left(\begin{array}{l}j \\ i\end{array}\right) a_{j}$. 
Remark 2.1. Note that $\boldsymbol{T}_{x}$ does not depend on the values of $g_{i, z}$, and that $\boldsymbol{t}_{x}$ can be calculated when $g_{z}$ is known for all $z<x$.

Proof: The expression (2.3) is obtained from the definition (2.1) of $g_{i, x}$ by noting that $f_{0}^{* n}=f_{0}^{n}$. Also the fact that $g_{i, x}=0$ for $x=1, \ldots, m-1$ is an immediate consequence of (2.1) since $f_{x}^{* n}=0$ for $x=1, \ldots, m-1$.

From De PRIL (1985) we have the identity,

$$
0=\sum_{y=0}^{x}\left[(n+1) \frac{y}{x}-1\right] f_{y} f_{x-y}^{* n} .
$$

Multiplying (2.7) by $p_{n} n^{i}$ and summing over $n \geq 0$ yields

$$
0=\sum_{y=0}^{x} f_{y}\left\{\frac{y}{x} g_{i+1, x-y}+\left(\frac{y}{x}-1\right) g_{i, x-y}\right\} .
$$

By omitting terms corresponding to $y=0, \ldots, m-1$ from the summation and substituting $x:=x-m$, we obtain after a little rearrangement that

$$
g_{i, x}-\frac{m}{x} g_{i+1, x}=t_{i, x},
$$

where $t_{i, x}$ is given by (2.5). From assumption (1.4) we obtain for $n \geq 1$ that

$$
p_{n} \sum_{i=0}^{k} b_{i} n^{i}=p_{n-1} \sum_{i=0}^{k} a_{i} n^{i}=p_{n-1} \sum_{i=0}^{k} c_{i}(n-1)^{i},
$$

where

$$
c_{i}=\sum_{j=i}^{k}\left(\begin{array}{l}
j \\
i
\end{array}\right) a_{j} .
$$

Multiplying (2.10) by $f_{x}^{* n}=\Sigma_{y=0}^{x} f_{y} f_{x-y}^{*(n-1)}$ and summing over $n \geq 1$ yields for $x \geq 1$ the relation

$$
\sum_{i=0}^{k} b_{i} g_{i, x}=\sum_{i=0}^{k} \sum_{y=0}^{x} f_{y} c_{i} g_{i, x-y}, \quad x \geq 1 .
$$

By isolating terms involving $g_{i, x}$ on the left-hand side, we rewrite (2.12) as

$$
\sum_{i=0}^{k}\left(b_{i}-f_{0} c_{i}\right) g_{i, x}=t_{k, x}, \quad x \geq 1,
$$

where $t_{k, x}$ is given by (2.6). The linear equations $\boldsymbol{T}_{x} \boldsymbol{g}_{x}=\boldsymbol{t}_{x}$, with $\boldsymbol{T}_{x}$ given by (2.4), now follow from (2.9) for $i=0, \ldots, k-1$ and (2.13).

QED

Remark 2.2. It is useful to consider separately the to cases where $m>0$ $\left(f_{0}=0\right)$ and $m=0\left(f_{0}>0\right)$. 
$m>0$. When $f_{0}=0$ we note from (2.3) that $g_{0,0}=p_{0}$ and $g_{i, 0}=0$ for $i \geq 1$. Note also that the terms $f_{0} c_{i}$ in the last row of $\boldsymbol{T}_{x}$ disappear in this case.

$m=0$. The linear equations $\boldsymbol{T}_{x} g_{x}=\boldsymbol{t}_{x}$ are easily solved analytically in this case, and we obtain that

$$
g_{i, x}=\frac{1}{f_{0}} \sum_{y=1}^{x} f_{y}\left\{\frac{y}{x} g_{i+1, x-y}+\frac{y-x}{x} g_{i, x-y}\right\}, \quad i<k,
$$

$$
g_{k, x}=\frac{1}{b_{k}-f_{0} c_{k}}\left\{\sum_{y=1}^{x} f_{y} \sum_{i=0}^{k} c_{i} g_{i, x-y}+\sum_{i=0}^{k-1}\left(f_{0} c_{i}-b_{i}\right) g_{i, x}\right\} \text {. }
$$

The initial values $g_{i, 0}$ may be expressed in terms of the derivatives $\varphi^{(j)}\left(f_{0}\right)$, $j=0, \ldots, k$, where $\varphi(\cdot)$ denotes the probability generating function of the counting distribution. However, for the class (1.4) of counting distributions, there is in general no simple expression for $\varphi(\cdot)$.

Example 1: The Waring distribution arises as a mixed geometric distribution with a beta mixing function. If $\mathrm{P}(N=n \mid \rho)=(1-\rho) \rho^{n}$, and $\rho \sim \operatorname{Beta}(\alpha, \beta)$, then

$$
p_{n}=\frac{\mathrm{B}(\alpha+n, \beta+1)}{\mathrm{B}(\alpha, \beta)},
$$

and

$$
p_{n}=\frac{n+\alpha-1}{n+\alpha+\beta} p_{n-1}
$$

This corresponds to (1.4) with $k=1$ and

$$
\begin{array}{ll}
a_{0}=\alpha-1 & a_{1}=1 \\
b_{0}=\alpha+\beta & b_{1}=1, \\
c_{0}=\alpha & c_{1}=1
\end{array}
$$

where $c_{i}$ is obtained from (2.11).

Example 2: For the hypergeometric distribution with parameters $(s, D, S)$,

$$
p_{n}=\frac{\left(\begin{array}{c}
D \\
n
\end{array}\right)\left(\begin{array}{c}
S-D \\
s-n
\end{array}\right)}{\left(\begin{array}{l}
S \\
s
\end{array}\right)}
$$


it holds that

$$
p_{n}=\frac{[n-(D+1)][n-(s+1)]}{n[n+(S-D-s)]} p_{n-1},
$$

which corresponds to (1.4) with $k=2$ and

$$
\begin{array}{lll}
a_{0}=(D+1)(s+1) & a_{1}=-(D+s+2) & a_{2}=1 \\
b_{0}=0 & b_{1}=S-D-s & b_{2}=1 . \\
c_{0}=D s & c_{1}=-(D+s) & c_{2}=1
\end{array}
$$

Example 3: The Polya-Eggenberger (Negative Hypergeometric) distribution arises as a mixed binomial distribution with a beta mixing function. The probability function

$$
p_{n}=\frac{\left(\begin{array}{c}
\alpha+n-1 \\
n
\end{array}\right)\left(\begin{array}{c}
\beta+M-n-1 \\
M-n
\end{array}\right)}{\left(\begin{array}{c}
\alpha+\beta+M-1 \\
M
\end{array}\right)}
$$

satisfies

$$
p_{n}=\frac{[n-(M+1)][n+(\alpha-1)]}{n[n-(M+\beta)]} p_{n-1},
$$

which corresponds to (1.4) with $k=2$ and

$$
\begin{array}{lll}
a_{0}=-(M+1)(\alpha-1) & a_{1}=-(M-\alpha+2) & a_{2}=1 \\
b_{0}=0 & b_{1}=-(M+\beta) & b_{2}=1 . \\
c_{0}=-M \alpha & c_{1}=(\alpha-M) & c_{2}=1
\end{array}
$$

Example 4: The generalized Waring distribution arises as a mixed negative binomial distribution with a beta mixing function,

$$
p_{n}=\frac{\Gamma(c+n)}{\Gamma(c) n !} \frac{\Gamma(\alpha+\beta)}{\Gamma(\alpha) \Gamma(\beta)} \frac{\Gamma(\alpha+n) \Gamma(\beta+c)}{\Gamma(\alpha+\beta+c+n)},
$$

and

$$
p_{n}=\frac{[n+(c-1)][n+(\alpha-1)]}{n[n+(\alpha+\beta+c-1)]} p_{n-1}
$$


This corresponds to (1.4) with $k=2$ and

$$
\begin{array}{lll}
a_{0}=(\alpha-1)(c-1) & a_{1}=(\alpha+c-2) & a_{2}=1 \\
b_{0}=0 & b_{1}=\alpha+\beta+c-1 & b_{2}=1 . \\
c_{0}=\alpha c & c_{1}=\alpha+c & c_{2}=1
\end{array}
$$

\section{RECURSIVE CALCULATION OF MOMENTS}

For the class (1.3) of counting distributions it was pointed out by DE PRIL (1986) that also the moments $m_{s}=\mathrm{E} X^{s}, s=0,1, \ldots$, of the compound distribution can be calculated recursively in a simple manner. Expressions for the moments $m_{s}$ are useful if one wants to calculate the NP- or Edgeworth approximation to the compound distribution as an alternative to the (exact) recursive method.

Let

$$
\mu_{s}=\mathrm{E} Y_{1}^{s}
$$

denote the $s^{\prime}$ th moment around the origin of the severity distribution, and define

$$
m_{i, s}=\sum_{x=0}^{\infty} x^{s} g_{i, x},
$$

where $g_{i, x}$ is the auxiliary function (2.1). Note in particular that $m_{s}=m_{0, s}$ is the $s$ 'th moment of the compound distribution. The following result gives a recursion for the vector $\left(m_{0, s}, \ldots, m_{k, s}\right), s=0,1, \ldots$, and hence the moments $m_{s}$.

Theorem 2: Assume that (1.4) holds true. With initial values

$$
m_{i, 0}=\mathrm{E} N^{i}=\sum_{n=0}^{\infty} p_{n} n^{i}, \quad i=0, \ldots, k,
$$

the moments $m_{s}=m_{0, s}$ of the compound distribution may be obtained by calculating $\left(m_{0, s}, \ldots, m_{k, s}\right)$ recursively for $s=1,2, \ldots$ as

$$
\begin{aligned}
& m_{i, s}=\sum_{j=0}^{s-1}\left(\begin{array}{c}
s-1 \\
j
\end{array}\right) \mu_{s-j} m_{i+1, j}-\sum_{j=1}^{s-1}\left(\begin{array}{c}
s-1 \\
j-1
\end{array}\right) \mu_{s-j} m_{i, j}, \quad i<k \\
& m_{k, s}=\frac{1}{b_{k}-c_{k}}\left[\sum_{i=0}^{k} c_{i} \sum_{j=0}^{s-1}\left(\begin{array}{l}
s \\
j
\end{array}\right) \mu_{s-j} m_{i, j}+\sum_{i=0}^{k-1}\left(c_{i}-b_{i}\right) m_{i, s}\right]
\end{aligned}
$$

Remark 3.1. When $\left(m_{0, u}, \ldots, m_{k, u}\right)$ is known for $u<s$, one calculates $m_{i, s}$ for $i=0, \ldots, k-1$ from (3.3) and then $m_{k, s}$ from (3.4). 
Proof: According to (3.1) and (2.1) the initial values are given by

$$
m_{i, 0}=\sum_{x=0}^{\infty} g_{i, x}=\sum_{x=0}^{\infty} \sum_{n=0}^{\infty} n^{i} p_{n} f_{x}^{* n}=\sum_{n=0}^{\infty} p_{n} n^{i}
$$

To verify (3.3) we multiply (2.8) by $x^{s}, s \geq 1$, and sum over $x \geq 0$ to obtain

$$
0=\sum_{x=0}^{\infty} \sum_{y=0}^{x} f_{y}\left\{y x^{s-1} g_{i+1, x-y}+\left(y x^{s-1}-x^{s}\right) g_{i, x-y}\right\} .
$$

By changing the order of summation and using the binomial formula

$$
x^{s}=\sum_{j=0}^{s}\left(\begin{array}{l}
s \\
j
\end{array}\right) y^{s-j}(x-y)^{j}
$$

(and the similar expression for $x^{s-1}$ ) it follows that

$$
\begin{aligned}
& 0=\sum_{y=0}^{\infty} f_{y}\left[y \sum_{j=0}^{s-1}\left(\begin{array}{c}
s-1 \\
j
\end{array}\right) y^{s-1-j}\left(m_{i+1, j}+m_{i, j}\right)-\sum_{j=0}^{s}\left(\begin{array}{l}
s \\
j
\end{array}\right) y^{s-j} m_{i, j}\right] \\
& =\sum_{j=0}^{s-1}\left(\begin{array}{c}
s-1 \\
j
\end{array}\right) \mu_{s-j}\left(m_{i+1, j}+m_{i, j}\right)-\sum_{j=0}^{s}\left(\begin{array}{l}
s \\
j
\end{array}\right) \mu_{s-j} m_{i, j} \text {. }
\end{aligned}
$$

Equation (3.3) now follows by extracting the term corresponding to $j=s$ from the last sum and making use of the fact that

$$
\left(\begin{array}{l}
s \\
j
\end{array}\right)=\left(\begin{array}{c}
s-1 \\
j
\end{array}\right)+\left(\begin{array}{c}
s-1 \\
j-1
\end{array}\right) .
$$

To verify (3.4), multiply (2.12) by $x^{s}, s \geq 1$, and sum over $x \geq 0$ to obtain

$$
\sum_{i=0}^{k} b_{i} m_{i, s}=\sum_{i=0}^{k} \sum_{x=0}^{\infty} \sum_{y=0}^{x} x^{s} f_{y} g_{i, x-y} c_{i} .
$$

By changing the order of summation and using (3.5), it follows that

$$
\begin{aligned}
\sum_{i=0}^{k} b_{i} m_{i, s} & =\sum_{i=0}^{k} \sum_{y=0}^{\infty} \sum_{j=0}^{s}\left(\begin{array}{l}
s \\
j
\end{array}\right) f_{y} c_{i} y^{s-j} m_{i, j} \\
& =\sum_{i=0}^{k} c_{i} \sum_{j=0}^{s}\left(\begin{array}{l}
s \\
j
\end{array}\right) \mu_{s-j} m_{i, j},
\end{aligned}
$$

and (3.4) follows by solving (3.6) for $m_{k, s}$. 
4. COMPaRison With the ReCURSIONS OF Willmot \& PANJER (1987)

In Panjer \& Willmot (1982) it is demonstrated how recursions for the compound distribution may be obtained by use of generating functions; in principle for arbitrary $k$ when the counting distribution satisfies (1.4). Formulas for the cases $k=1$ and $k=2$ are found in Willmot \& PANJER (1987). We cite the following recursive procedure:

Define the auxiliary function

$$
\begin{aligned}
& q_{0}=m, \\
& q_{x}=\frac{(x+m) f_{x+m}}{f_{m}}-\sum_{y=1}^{x} \frac{f_{y+m}}{f_{m}} q_{x-y},
\end{aligned}
$$

where $m$ is the smallest integer such that $f_{m}>0$, and also

$$
\begin{aligned}
& t_{0}=r-1, \\
& t_{x}=\frac{(x+r)(x+r-1) f_{x+r}}{r f_{r}}-\sum_{y=1}^{x} \frac{(y+r) f_{y+r}}{r f_{r}} t_{x-y},
\end{aligned}
$$

where $r$ is the smallest integer such that $r f_{r}>0$.

For $k=1$ the class (1.4) may be rewritten as

$$
p_{n}=\frac{\beta(n-1)+\kappa}{\alpha n+1} p_{n-1}, \quad n=1,2, \ldots,
$$

and the compound distribution $g_{x}$ satisfies the recursion

$$
=\frac{p_{0} q_{x}+\sum_{y=1}^{x}\left[(\beta(y-x)+\kappa y) f_{y}-q_{y}\right] g_{x-y}}{x\left(\alpha-\beta f_{0}\right)+q_{0}} .
$$

For $k=2$ and $b_{0}=0$ we may rewrite (1.4) as,

$$
p_{n}=\frac{\beta(n-1)(n-2)+\kappa(n-1)+\delta}{n(n-1)+\alpha n} p_{n-1}, \quad n=1,2, \ldots
$$

Define a new set of auxiliary functions,

$$
u_{x}=\sum_{y=0}^{x} y q_{x-y} f_{y}, \quad v_{x}=\sum_{y=0}^{x} t_{x-y} f_{y}
$$

and $g_{x}$ can be calculated recursively as

$$
g_{x}=\frac{\sum_{y=1}^{x} g_{x-y} k_{x, y}}{x\left[\left(x-t_{0}-1\right)\left(1-\beta f_{0}\right)+\alpha q_{0}\right]},
$$


where

$$
k_{x, y}=(x-y)\left\{t_{y}-\alpha q_{y}-\beta v_{y}+[\kappa y+\beta(x-y-1)] f_{y}\right\}+\delta u_{y} .
$$

It is interesting to compare the recursions (4.1)-(4.6) to the one proposed in Theorem 1.

Each step in the proposed recursion involves $(k+1)$ summations of the type $\sum_{y=1}^{x} f_{y} h_{x, y}$ (for some function $h_{x, y}$ ). The number of computations involved with the calculation of $g_{x}$ when $g_{0}, \ldots, g_{x-1}$ are known is therefore proportional to $x$, and the number of computations involved with $g_{x}$ is of order $x^{2}$. In practice, the severity distribution $f_{y}$ has finite support such that $f_{y}=0$ for $y>y_{\max }$, say. In this case the sum $\Sigma_{y=1}^{x} f_{y} h_{x, y}$ involves only $y_{\max }$ non-zero terms, and the number of computations involved with $g_{x}$ is of order $x$.

TABLE 1

COMPUTING TIME, minutes: seconds TO OBTAIN $g_{x}$ FOR $k=2$ WHEN $f_{y}$ HAS FINITE SUPPORT WITH $y_{\max }=50$

\begin{tabular}{c|c|c|c}
\hline \hline$x$ & $m>0$ & $m=0$ & Willmot \& Panjer \\
\hline 200 & $0: 04$ & $0: 04$ & $0: 07$ \\
400 & $0: 09$ & $0: 08$ & $0: 22$ \\
600 & $0: 14$ & $0: 13$ & $0: 45$ \\
800 & $0: 20$ & $0: 19$ & $1: 16$ \\
1000 & $0: 26$ & $0: 24$ & $1: 54$ \\
1200 & $0: 32$ & $0: 30$ & $2: 41$ \\
1400 & $0: 39$ & $0: 37$ & $3: 37$ \\
1600 & $0: 46$ & $0: 44$ & $5: 43$ \\
1800 & $0: 54$ & $0: 51$ & $7: 18$ \\
2000 & $1: 02$ & $0: 59$ & \\
\hline
\end{tabular}

Also the recursions (4.3) and (4.5) of Willmot \& PANJER (1987) involve summations $\Sigma_{y=1}^{x}$. However, these sums do not simplify in the case where $f_{y}$ has finite support, and the total number of computations is therefore of order $x^{2}$.

Table 1 shows for $k=2$ the total computing time as a function of $x$ for the recursion of Willmot \& PANJER (1987) and for the proposed recursion. For the latter, we have treated separately the two cases where $m>0$ and $m=0$ (see Remark 2.2). In the first case we have programmed the recursion as presented in Theorem 1, and the matrix $\boldsymbol{T}_{x}$ has been inverted using STSC APL standard facilities. In the latter case we have used the formulas (2.14) and (2.15). The computations were done on a $486,50 \mathrm{mHz}$ PC. The severity distribution has been chosen such that $y_{\max }=50$. It should be noted that the computing time does not depend on the actual choice of parameters for the counting distribution, and also not on the actual choice of severity distribution (except for the choice of $y_{\max }$ ). The results are also displayed in Figure 1, where the computing times (in seconds) are shown as a function of $x$. It is seen that the 


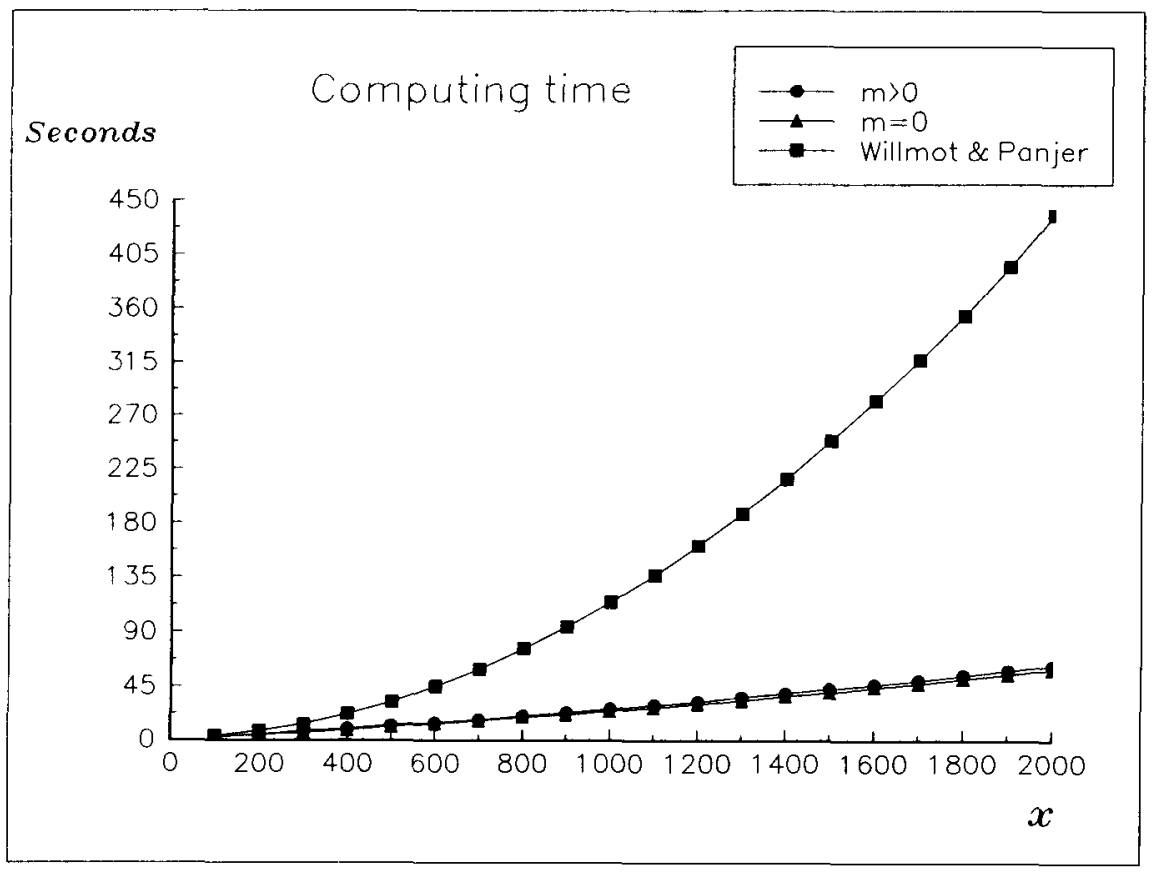

Figure 1. Computing time to obtain $g_{x}$ for $k=2$ when $f_{y}$ has finite support with $y_{\max }=50$.

total computing time is linear in $x$ for the proposed recursion and quadratic for the recursion of WILLMOT \& PANJER (1987).

With a hypergeometric counting distribution $(k=2)$ we have checked the recursions for numerical instabilities. We consider two different severity distributions,

$$
\begin{aligned}
& f_{1, y}=e^{-3 y} / \sum_{y=0}^{20} e^{-3 y}, \quad y=0, \ldots, 20 . \\
& f_{2, y}=1 / 150, \quad y=0, \ldots, 149 .
\end{aligned}
$$

The distribution $f_{1}$ is very short-tailed with a high probability $f_{0}=0.2837$ of zero-claims. The second distribution $f_{2}$ is more heavy-tailed with a "large" average claim size $\mathrm{E} Y=74.5$. For each of the severity distributions $f_{1}$ and $f_{2}$ we have calculated the compound distribution using a hypergeometric counting distribution with parameters $(s, D, S)$ (see Example 2), where $D=S / 4$ and $s=q S$, and $(S, q)$ varies in the set $\{40,100,200\} \times\{0.25,0.5,0.75\}$. The corresponding average number of claims, $\mathrm{EN}=s D / S=q S / 4$ is shown in Table 2. For the proposed recursion, $m>0$, we have shifted the distributions $f_{1}$ and $f_{2}$ one step to the right, such that $m=1$ in this case. The check for numerical instabilities was performed by simple graphical inspection. In Table 3 we have indicated by a $*$ those cases where instabilities were found. All computations were continued until the $99.5 \%$ fractile of the compound distributions was reached. 
TABLE 2

Average number of Claims, EN $=q S / 4$

\begin{tabular}{l|c|c|c}
\hline \hline & $q=0.25$ & $q=0.5$ & $q=0.75$ \\
\hline$S=40$ & 2.5 & 5 & 7.5 \\
$S=100$ & 6.25 & 12.5 & 18.75 \\
$S=200$ & 12.5 & 25 & 37.5 \\
\hline
\end{tabular}

TABLE 3

NUMERICAL INSTABILITIES FOR COMPOUND HYPERGEOMETRIC DISTRIBUTIONS. INSTABILITIES ARE INDICATED BY A *

\begin{tabular}{l|c|c|c|c|c|c}
\hline \hline \multirow{2}{*}{} & \multicolumn{5}{|c|}{ Severity distribution $f_{1}$} & \multicolumn{4}{c}{ Severity distribution $f_{2}$} \\
\cline { 2 - 7 } & $q=0.25$ & $q=0.5$ & $q=0.75$ & $q=0.25$ & $q=0.5$ & $q=0.75$ \\
\hline$S=40$ & & & & \multicolumn{5}{|c|}{$m>0$} & & \\
\hline$S=100$ & & & & & & \\
\hline$S=200$ & & & & & & \\
\hline
\end{tabular}

\begin{tabular}{l|c|c|c|c|c|c}
\hline & \multicolumn{5}{|c}{$m=0$} & \\
\hline$S=40$ & & & & $*$ & & \\
\hline$S=100$ & & & & $*$ & $*$ & \\
\hline$S=200$ & $*$ & $*$ & & $*$ & $*$ & \\
\hline
\end{tabular}

\begin{tabular}{l|l|l|l|l|l|c}
\hline & \multicolumn{5}{|c}{ Willmot \& Panjer } \\
\hline$S=40$ & & & & & & \\
\hline$S=100$ & & & & & $*$ & $*$ \\
\hline$S=200$ & & & & $*$ & $*$ & $*$ \\
\hline
\end{tabular}

It is noted that no instabilities were found for the proposed recursion in the case where $m>0$. The recursion of Willmot \& PANJER (1987) was unstable for the severity distribution $f_{2}$, when the average number of claims exceeds 10 (in this case). These instabilities can be attributed the accumulation of round-off errors. The proposed recursion, when $m>0$, was unstable for "large" values of $S$ and "small" values of $q$-irrespective of which severity distribution was used. An explanation for this instability can be found by examining the expression for $g_{k, x}$ in (2.15). This expression involves subtraction of terms $b_{i} g_{i, x}, i<k$, and subtraction (of equally large numbers) is known to 
increase the relative errors. For the hypergeometric distribution it holds that $b_{0}=0$ and $b_{2}=1$, whereas $b_{1}=S-D-s$ (see Example 2). For the present combination of parameters it holds that $b_{1}=S(0.75-q)$, which assumes its maximum when $S$ is "large" and $q$ is "small". In general, we would therefore expect that the proposed recursion is unstable for $m=0$ when $S-D \gg s$ and stable when $S-D \approx s$.

It should be noted that all calculations were done with single precision, and that the results could (obviously) be improved by using double precision.

\section{CALCULATION OF RUIN PROBABILITIES}

Let

$$
S(t)=\sum_{i=1}^{N(t)} Z_{i}
$$

where $N(t)$ denotes the number of claims incurred during $[0, t]$, and $Z_{1}, Z_{2}, \ldots$, denote the corresponding claim amounts. The amounts $Z_{i}$ are assumed to be independent of $N(t)$ and mutually independent with common distribution $H$. The average claim size is denoted by $\mu=\mathrm{E} Z_{1}$.

If premiums are paid continuously at a rate $B$ pr. time unit, the maximal loss incurred is

$$
L=\sup _{t \geq 0}\{S(t)-B t\},
$$

and the probability of ultimate ruin is

$$
\psi(u)=\mathrm{P}(L>u),
$$

where $u$ denotes the initial capital. Assume that $B=(1+\theta) \lambda \mu$, where the relative safety loading $\theta$ is non-negative. It is a well known result (see e.g. Bowers et al., 1986) that if $\{N(t)\}$ is a time-homogeneous Poisson process with claims rate $\lambda$, then

$$
L \stackrel{D}{=} \sum_{i=0}^{M} L_{i}
$$

where $M$ has a geometric distribution

$$
\mathbf{P}(M=m)=(1-\rho) \rho^{m}, \quad \rho=\frac{1}{1+\theta}, \quad m=0,1, \ldots,
$$

and $L_{1}, L_{2}, \ldots$ are mutually independent with common density

$$
f(y)=(1-H(y)) / \mu .
$$

Panjer (1986) suggested a discrete approximation to $f(y)$, and then to calculate $\psi(u)$ recursively by means of the Panjer-recursion, which is valid in the case of geometric counting distributions. 
Consider now the case where $\{N(t)\}$, conditionally given $A=\lambda$, is a Poisson process with claims rate $\lambda$. Since, in this case,

$$
(L \mid \Lambda=\lambda) \stackrel{D}{=} \sum_{i=0}^{M} L_{i}
$$

with $M$ and $L_{i}$ being distributed as before, it follows that

$$
L \stackrel{D}{=} \sum_{i=0}^{M^{\prime}} L_{i}
$$

where $L_{i}$ still is distributed according to (5.3), and $M^{\prime}$ has a mixed geometric distribution. If we take a beta mixing function with parameters $(\alpha, \beta)$ for $\rho$ appearing in (5.2), it follows that $M^{\prime}$ has the Waring distribution from Example 1. Using the same method as suggested by PANJER (1986) for discretizing the density (5.3), we may then apply Theorem 1 with $k=1$ to obtain a recursive method for calculating $\psi(u)$.

Note, that if $\rho$ is beta distributed with parameters $(\alpha, \beta)$, then the claims rate $\Lambda$ is distributed as $(B / \mu) U$, where $U$ is beta distributed with parameters $(\alpha, \beta)$.

\section{REFERENCES}

Bowers, N. L. et al., (1986) Actuarial Mathematics. The Society of Actuaries, Itasca, Illinois.

DE PRIL, N. (1985) Recursions for convolutions of arithmetic distributions. ASTIN Bulletin 15, $135-139$.

DE PRIL, N. (1986) Moments of a class of compound distributions. Scand. Actuarial J. 1986, $117-120$.

GuldBerg, A. (1931) On discontinuous frequency-functions and statistical series. Skandinavisk Aktuarietidskrift 14, 167-187.

ORD, J.K. (1967). On a system of discrete distributions. Biometrika 54, 649656.

Panjer, H.H. (1981) Recursive evaluation of a family of compound distributions. ASTIN Bulletin 11, $22-26$.

Panjer, H. H. (1986) Direct calculation of ruin probabilities. The Journ. of Risk and Insurance LIII, $521-529$.

Panjer, H.H. and Willmot, G. E. (1982) Recursions for compound distributions. ASTIN Bulletin $13,1-11$.

Panjer, H.H. and Willmot, G.E. (1986) Computational aspects of recursive evaluation of compound distributions. Insurance: Math. and Econ. 5, 113-116.

SCHRÖTER, K.J. (1990) On a family of counting distributions and recursions for related compound distributions. Scand. Actuarial $J$ 1990, 161-174.

SundT, B. (1992) On some extensions of Panjer's class of counting distributions. ASTIN Bulletin 22, 61-80.

SundT, B. and Jewell, W.S. (1981) Further results on recursive evaluation of compound distributions. ASTIN Bulletin 11, 27-39.

Willmot, G.E. and PANJer, H.H. (1987) Difference equation approaches in evaluation of compound distributions. Insurance: Math. and Econ. 6, 43-56.

\section{Ole Hesselager}

Laboratory of Actuarial Mathematics, Universiteitsparken 5, University of Copenhagen, DK-2100 Copenhagen $\emptyset$. 